MILITARY TECHNICAL COLLEGE CAIRO - EGYPT

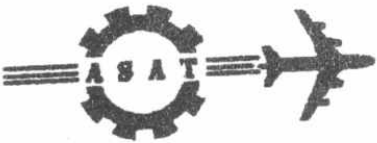

$7^{\text {th }}$ INTERNATIONAL CONF. ON AEROSPACE SCIENCES \& AVIATION TECHNOLOGY \title{
NUMERICAL SOLUTION UF VORTICITY TRANSPORT EQUATIONS IN TERMS OF
THREE-DIMENSIONAL STREAM FUNCTION VECTOR COMPONENTS
}

\author{
A. M. ELSHABKA* and T. J. CHUNG**
}

\begin{abstract}
The fourth order three component partial differential equations of vorticity transport in terms of three-dimensional stream function vector components are formulated. The generalized Galerkin finite element solutions of the governing equations for incompressible flow is then carried out, and the results are shown to be very accurate for the lid-driven three-dimensional cavity problems examined herein. This numerical accuracy is attributed to the correct definition of threedimensional stream function vector components, appropriate finite element interpolation functions, associated physically simple boundary conditions, and the rigorous Newton-Raphson scheme of solution process, as well as the governing equations being free from pressure oscillations. To the best of our knowledge, the present study marks the first attempt to solve the three-dimensional fourth order partial differential equations of vorticity transport for the three-dimensional stream function vector components.
\end{abstract}

\section{KEYWORDS}

Vorticity transport equations / Three-dimensional stream function vector / Three-dimensional finite element formulation / Three-dimensional interpolation function

\section{INTRODUCTION}

The precise theoretical arguments for the stream function in three dimensions are important when the momentum equations are written in terms of stream function(s), leading to the fourth order partial differential equations. In two dimensions, the two momentum equations result in a single biharmonic equation in terms of a single scalar stream function. Obviously, this is not the case in three dimensions. This is because we must deal with streamlines parallel to each of the three components of the three-dimensional velocity vector. Thus, it is necessary that the stream function be expanded into three components with each component corresponding to each of the three velocity components. The existence of three-dimensional stream function vector $\Psi$ associated with the three-dimensional velocity vector have been justified [1-3].

* Ph.D. Aeronautical Department, Military Technical College, Cairo, Egypt.

** Prof. Department of Mechanical and Aerospace Engineering, The University of Alabama in Huntsville, Huntsville, AL 35899, USA. 
with

$$
\mathbf{V}=\nabla \psi \times \hat{\mathbf{n}}=\nabla \times \Psi=\varepsilon_{\mathrm{ijk}} \Psi_{\mathrm{k}, \mathrm{j}} \mathrm{i}_{\mathrm{i}}
$$

$$
\Psi=\psi \hat{\mathbf{n}}
$$

$\psi$...is a scalar stream function and $\hat{\mathbf{n}}$...is a unit normal vector.

The stream function vector $\Psi$ and its components $\Psi_{\mathrm{i}}$ described on each surface designated by $\hat{\mathrm{n}}_{\mathrm{i}}$ as (see Fig. lb):

with

$$
\Psi=\Psi_{\mathrm{i}} \mathrm{i}_{\mathrm{i}} \quad(\mathrm{i}=1,2,3)
$$

$\Psi_{1}=\psi \hat{\mathrm{n}}_{1}, \Psi_{2}=\psi \hat{\mathrm{n}}_{2}$, and $\Psi_{3}=\psi \hat{\mathrm{n}}_{3}$

Note that Eqs. (1) satisfies identically the conservation of mass in steady state and reduce automatically to the two-dimensional incompressible flow in terms of a single stream function $\psi=\Psi_{3}$

$$
\begin{array}{ll}
\mathrm{u}=\frac{\partial \psi}{\partial \mathrm{y}} \hat{\mathrm{n}}_{3}=\frac{\partial \psi}{\partial \mathrm{y}} & \left(\hat{\mathrm{n}}_{3}=1 \text { on } \hat{\mathrm{n}}_{3} \text { surface }\right) \\
v=-\frac{\partial \psi}{\partial \mathrm{x}} \hat{\mathrm{n}}_{3}=-\frac{\partial \psi}{\partial \mathrm{x}} & \left(\hat{\mathrm{n}}_{3}=1 \text { on } \hat{\mathrm{n}}_{3} \text { surface }\right)
\end{array}
$$

The vorticity vector assumes the form

$$
\omega=\nabla \times \mathbf{V}=\nabla \times(\nabla \times \Psi)=\nabla(\nabla \cdot \Psi)-\nabla^{2} \Psi=-\nabla^{2} \Psi
$$

where $(\nabla \cdot \Psi)=0$ arises simply from the geometrical property, $\nabla \psi \cdot \hat{\mathbf{n}}=0$ in Fig. 1a. Thus, an irrotational ideal flow in three-dimensions can be given by $\nabla^{2} \Psi=0$ in terms of three-dimensional stream function vector.

In the past, solutions of three-dimensional incompressible flows were obtained using the second order partial differential equations of momentum with either velocity-pressure formulation or velocity-vorticity formulation. In the former, the pressure Poisson equation is solved, assuring the conservation of mass. In the latter, however, the pressure gradient terms do not explicitly arise in the momentum equation, and the mass conservation is automatically satisfied in the process of the derivation of the vorticity transport equation.

In this paper, we discuss the finite element solution of the fourth order partial differential equations derived from the three momentum equations in terms of the three-dimensional stream function vector components. First of all, appropriate interpolation functions for the variables must be constructed. The variables consist of not only the stream function vector components but also their derivatives. This is followed by the tensorial manipulations of Newton-Raphson derivatives and finally iterative solutions of the resulting algebraic equations. Accurate solutions have been obtained in [2], which are due to the correct definition of three-dimensional stream function vector components, associated physically simple boundary conditions, and the rigorous Newton-Raphson scheme of solution process. 
The fourth order partial differential equations of vorticity transport and the finite element interpolation functions are elaborated in section 2 , followed by the computational procedure in section 3 and the computational results in section 4 .

\section{MATHEMATICAL FORMULATION}

\subsection{Governing Equations}

For three-dimensional fluid flow with constant property, the conservation laws for mass and momentum can be expressed as:

$$
\begin{aligned}
& \frac{\partial \rho}{\partial \mathrm{t}}+\nabla \cdot(\rho \mathbf{V})=0 \\
& \rho \frac{\partial \mathbf{V}}{\partial \mathrm{t}}+\rho(\mathbf{V} \cdot \nabla) \mathbf{V}=-\nabla \mathrm{p}+\mu\left(\nabla^{2} \mathbf{V}+\frac{1}{3} \nabla(\nabla \cdot \mathbf{V})\right)+\rho \mathbf{F}
\end{aligned}
$$

where $\rho$ is the density; $\mathrm{p}$, the instantaneous static pressure; $\mathbf{V}$, the velocity vector; $\mathbf{F}$, the body force vector; and $\mu$ is the coefficient of dynamic viscosity.

Taking the curl of the momentum equation (8), we get the vorticity transport equation as:

$$
\frac{\partial_{0}}{\partial \mathrm{t}}-(\boldsymbol{\omega} \cdot \nabla) \mathbf{V}+(\mathbf{V} \cdot \nabla) \omega+(\nabla \cdot \mathbf{V}) \omega=\nu \nabla^{2} \boldsymbol{\omega}
$$

where $\omega$ is the vorticity vector given by equation (6).

For incompressible flow, Eqs. (7) and (9) take the form:

$$
\begin{aligned}
& \nabla \cdot \mathbf{V}=0 \\
& \frac{\partial \omega}{\partial \mathrm{t}}-(\omega \cdot \nabla) \mathbf{V}+(\mathbf{V} \cdot \nabla) \omega=\nu \nabla^{2} \omega
\end{aligned}
$$

Substituting Eqs. (1) and (6) into equation (11) leads to:

$$
\frac{\partial}{\partial t} \nabla^{2} \Psi-\left(\nabla^{2} \Psi \cdot \nabla\right)(\nabla \times \Psi)+((\nabla \times \Psi) \cdot \nabla) \nabla^{2} \Psi=\nu \nabla^{4} \Psi
$$

This is a fourth-order vector partial differential equation in three unknowns $\Psi_{1}, \Psi_{2}$, and $\Psi_{3}$. Obviously, this set of governing equations has two main advantages compared with that in terms of primitive variables. The continuity equation is satisfied by the three-dimensional stream function vector independently from other variables, and the pressure is eliminated from the system. The solution of equation (12) represents the first attempt of attacking the fourth order partial differential equations in terms of three-dimensional stream function vector components, as defined above. Details of the numerical solution can be found in [2]. 


\subsection{New Three-Dimensional Interpolation Functions}

To solve numerically the governing equations (12) by the finite element method, it is first necessary to derive the consistent interpolation functions for an eight node hexahedral element. In general these functions have to satisfy the following conditions:

1- The polynomial must be of the degree at least $n-1$, where $n$ is the order of the highest derivative in the finite element equation. This will assure the continuity of the dependent variables and its normal derivatives.

2- When the number of elements increases, the derivatives within the finite element equation will tend to have a constant, or in particular a zero value. Hence it is necessary to include, in our function, terms that represent these conditions.

For equation (12), the highest-order derivatives in the finite element equations are $\frac{\partial^{3} \Psi_{i}}{\partial x^{3}}, \frac{\partial^{3} \Psi_{i}}{\partial y^{3}}, \frac{\partial^{3} \Psi_{i}}{\partial z^{3}}, \frac{\partial^{3} \Psi_{i}}{\partial x^{2} \partial y}, \frac{\partial^{3} \Psi_{i}}{\partial y^{2} \partial x}, \frac{\partial^{3} \Psi_{i}}{\partial x^{2} \partial z}, \frac{\partial^{3} \Psi_{i}}{\partial z^{2} \partial x}, \frac{\partial^{3} \Psi_{i}}{\partial y^{2} \partial z}$, and $\frac{\partial^{3} \Psi_{i}}{\partial z^{2} \partial y}$. Hence the $\Psi_{\mathrm{i}}$ functions must have the following terms:

$$
\begin{gathered}
\Psi_{\mathrm{i}}=\alpha_{1}+\alpha_{2} \mathrm{x}+\alpha_{3} \mathrm{y}+\alpha_{4} \mathrm{z}+\alpha_{5} \mathrm{x}^{2}+\alpha_{6} \mathrm{xy}+\alpha_{7} \mathrm{yz}+\alpha_{8} \mathrm{xz}+ \\
\alpha_{9} \mathrm{y}^{2}+\alpha_{10} \mathrm{x}^{3}+\alpha_{11} \mathrm{x}^{2} \mathrm{y}+\alpha_{12} \mathrm{z}^{2}+\alpha_{13} \mathrm{x}^{2} \mathrm{z}+\alpha_{14} \mathrm{xy}^{2}+ \\
\alpha_{15} \mathrm{yz}^{2}+\alpha_{16} \mathrm{y}^{2} \mathrm{z}+\alpha_{17} \mathrm{xz}^{2}+\alpha_{18} \mathrm{xyz}+\alpha_{19} \mathrm{y}^{3}+\alpha_{20} \mathrm{z}^{3}
\end{gathered}
$$

The simplest hexahedral element (brick) has nodes at the eight corners and four variables per node as shown in Fig. 2. We take the nodal variable listing as:

$$
\Psi=\left\{\Psi_{1}, \frac{\partial \Psi_{1}}{\partial \mathrm{x}}, \frac{\partial \Psi_{1}}{\partial \mathrm{y}}, \frac{\partial \Psi_{1}}{\partial z}, \cdots \frac{\partial \Psi_{8}}{\partial z}\right\}
$$

Inter-element compatibility requires continuity of $\Psi$, and its normal derivative. Since only the first derivatives are included in equation (14), the expansion for $\Psi$ along a face must be cubic, to satisfy continuity of $\Psi$. Also, the normal derivatives must vary linearly to satisfy continuity of $\frac{\partial \Psi}{\partial \mathrm{n}_{\mathrm{i}}}$. The expansion consists of a complete cubic, equation (13), and twelve additional terms. We can choose the terms arbitrarily but we should preserve symmetry of the expansion to ensure invariance of the element matrices. The type of element used is based on the choice of:

$$
\left(x^{3} y, x y^{3}, y^{3} z, x^{3} z, y z^{3}, x z^{3}, x y^{2} z, x^{2} y z, x y z^{2}, x y^{3} z, x^{3} y z, x y z^{3}\right)
$$

The complete expansion is written in the nondimensional coordinate system $(\xi, \eta, \zeta)$ as:

$$
\begin{aligned}
\Psi_{\mathrm{i}}= & \alpha_{1}+\alpha_{2} \xi+\alpha_{3} \eta+\alpha_{4} \zeta+\alpha_{5} \xi^{2}+\alpha_{6} \xi \eta+\alpha_{7} \eta \zeta+\alpha_{8} \xi \zeta+\alpha_{9} \eta^{2} \\
& +\alpha_{10} \xi^{3}+\alpha_{11} \xi^{2} \eta+\alpha_{12} \zeta^{2}+\alpha_{13} \xi^{2} \zeta+\alpha_{14} \xi \eta^{2}+\alpha_{15} \eta \zeta^{2}+\alpha_{16} \eta^{2} \zeta \\
& +\alpha_{17} \xi \zeta^{2}+\alpha_{18} \xi \eta \zeta+\alpha_{19} \eta^{3}+\alpha_{20} \zeta^{3}+\alpha_{21} \xi^{3} \eta+\alpha_{22} \xi \eta^{3}+\alpha_{23} \eta^{3} \zeta \\
& +\alpha_{24} \xi^{3} \zeta+\alpha_{25} \eta \zeta^{3}+\alpha_{26} \xi \zeta^{3}+\alpha_{27} \xi \eta^{2} \zeta+\alpha_{28} \xi^{2} \eta \zeta+\alpha_{29} \xi \eta \zeta^{2} \\
& +\alpha_{30} \xi \eta^{3} \zeta+\alpha_{31} \xi^{3} \eta \zeta+\alpha_{32} \xi \eta \zeta^{3}
\end{aligned}
$$


Differentiating equation (15) with respect to $\xi, \eta$, and $\zeta$, we get:

$$
\begin{aligned}
\Psi_{\mathrm{i}, \xi}= & \alpha_{2}+2 \alpha_{5} \xi+\alpha_{6} \xi+\alpha_{8} \xi+3 \alpha_{10} \xi^{2}+2 \alpha_{11} \xi \eta+2 \alpha_{13} \xi \zeta+\alpha_{14} \eta^{2} \\
& +\alpha_{17} \zeta^{2}+\alpha_{18} \eta \zeta+3 \alpha_{21} \xi^{2} \eta+\alpha_{22} \eta^{3}+3 \alpha_{24} \xi^{2} \zeta+\alpha_{26} \zeta^{3}+\alpha_{27} \eta^{2} \zeta \\
& +2 \alpha_{28} \xi \eta \zeta+\alpha_{29} \eta \zeta^{2}+\alpha_{30} \eta^{3} \zeta+3 \alpha_{31} \xi^{2} \eta \zeta+\alpha_{32} \eta \zeta^{3} \\
\Psi_{\mathrm{i}, \eta}= & \alpha_{3}+\alpha_{6} \xi+\alpha_{7} \zeta+2 \alpha_{9} \eta+\alpha_{11} \xi^{2}+2 \alpha_{14} \xi \eta+\alpha_{15} \zeta^{2}+2 \alpha_{16} \eta \zeta \\
& +\alpha_{18} \xi \zeta+3 \alpha_{19} \eta^{2}+\alpha_{21} \xi^{3}+3 \alpha_{22} \xi \eta^{2}+3 \alpha_{23} \eta^{2} \zeta+\alpha_{25} \zeta^{3}+2 \alpha_{27} \xi \eta \zeta \\
& +\alpha_{28} \xi^{2} \zeta+\alpha_{29} \xi \xi^{2}+3 \alpha_{30} \xi \eta^{2} \zeta+\alpha_{31} \xi^{3} \zeta+\alpha_{32} \xi \zeta^{3} \\
\Psi_{i, \zeta}= & \alpha_{4}+\alpha_{7} \eta+\alpha_{8} \xi+2 \alpha_{12} \zeta+\alpha_{13} \xi^{2}+2 \alpha_{15} \eta \zeta+\alpha_{16} \eta^{2}+2 \alpha_{17} \xi \zeta \\
& +\alpha_{18} \xi \eta+3 \alpha_{20} \zeta^{2}+\alpha_{23} \eta^{3}+\alpha_{24} \xi^{3}+3 \alpha_{25} \eta \zeta^{2}+3 \alpha_{26} \xi \zeta^{2}+\alpha_{27} \xi \eta^{2} \\
& +\alpha_{28} \xi^{2} \eta+2 \alpha_{29} \xi \eta \zeta+\alpha_{30} \xi \eta^{3}+\alpha_{31} \xi^{3} \eta+3 \alpha_{32} \xi \eta \zeta^{2}
\end{aligned}
$$

where $\alpha_{1} \sim \alpha_{32}$ are unknown coefficients to be determined. Writing Eqs. (15-18) in terms of the nodal values, shown in Fig. 2, yields

$$
[\Psi]=[C][\alpha]
$$

where $[\mathrm{C}]$ is a coefficient matrix. Thus

$$
[\alpha]=[C]^{-1}[\Psi]
$$

Substituting equation (20) into Eqs.(15) via (18) yields

$$
\Psi_{i}=N_{j}^{0}\left(\Psi_{i}\right)_{j}+N_{i}^{1}\left(\frac{\partial \Psi_{i}}{\partial \xi}\right)_{j}+N_{j}^{2}\left(\frac{\partial \Psi_{i}}{\partial \eta}\right)_{j}+N_{j}^{3}\left(\frac{\partial \Psi_{i}}{\partial \zeta}\right)_{j}
$$

where the subscript $\mathrm{j}$ denotes element node number $(\mathrm{j}=1,2, \ldots 8)$. The functions $\mathrm{N}_{\mathrm{j}}^{0}, \mathrm{~N}_{\mathrm{j}}^{1}$, $\mathrm{N}_{\mathrm{j}}^{2}$, and $\mathrm{N}_{j}^{3}$ have been proven numerically to satisfy the following relations:

$$
\begin{aligned}
& \mathrm{N}_{\mathrm{j}}^{0}\left(\xi_{\mathrm{k}}, \eta_{\mathrm{k}}, \zeta_{\mathrm{k}}\right)=\delta_{\mathrm{jk}} \quad, \quad \mathrm{N}_{\mathrm{j}}^{1}\left(\xi_{\mathrm{k}}, \eta_{\mathrm{k}}, \zeta_{\mathrm{k}}\right)=0, \\
& \mathrm{~N}_{\mathrm{j}}^{2}\left(\xi_{\mathrm{k}}, \eta_{\mathrm{k}}, \zeta_{\mathrm{k}}\right)=0 \quad, \quad \mathrm{~N}_{\mathrm{j}}^{3}\left(\xi_{\mathrm{k}}, \eta_{\mathrm{k}}, \zeta_{\mathrm{k}}\right)=0, \\
& \frac{\partial}{\partial \xi}\left[\mathrm{N}_{j}^{0}\left(\xi_{k}, \eta_{k}, \zeta_{k}\right)\right]=0 \quad, \quad \frac{\partial}{\partial \xi}\left[\mathrm{N}_{j}^{1}\left(\xi_{k}, \eta_{k}, \zeta_{k}\right)\right]=\delta_{j k}, \\
& \frac{\partial}{\partial \xi}\left[\mathrm{N}_{j}^{2}\left(\xi_{k}, \eta_{k}, \zeta_{k}\right)\right]=0 \quad, \quad \frac{\partial}{\partial \xi}\left[\mathrm{N}_{j}^{3}\left(\xi_{k}, \eta_{k}, \zeta_{k}\right)\right]=0 \quad \text {, } \\
& \frac{\partial}{\partial \eta}\left[\mathrm{N}_{\mathrm{j}}^{0}\left(\xi_{\mathrm{k}}, \eta_{\mathrm{k}}, \zeta_{\mathrm{k}}\right)\right]=0 \quad, \quad \frac{\partial}{\partial \eta}\left[\mathrm{N}_{\mathrm{j}}^{1}\left(\xi_{\mathrm{k}}, \eta_{\mathrm{k}}, \zeta_{\mathrm{k}}\right)\right]=0,
\end{aligned}
$$




$$
\begin{array}{lll}
\frac{\partial}{\partial \eta}\left[\mathrm{N}_{j}^{2}\left(\xi_{k}, \eta_{k}, \zeta_{k}\right)\right]=\delta_{j k} & , & \frac{\partial}{\partial \eta}\left[\mathrm{N}_{j}^{3}\left(\xi_{k}, \eta_{k}, \zeta_{k}\right)\right]=0, \\
\frac{\partial}{\partial \zeta}\left[\mathrm{N}_{j}^{0}\left(\xi_{k}, \eta_{k}, \zeta_{k}\right)\right]=0 & , & \frac{\partial}{\partial \zeta}\left[\mathrm{N}_{j}^{1}\left(\xi_{k}, \eta_{k}, \zeta_{k}\right)\right]=0, \\
\frac{\partial}{\partial \zeta}\left[\mathrm{N}_{j}^{2}\left(\xi_{k}, \eta_{k}, \zeta_{k}\right)\right]=0 & \frac{\partial}{\partial \zeta}\left[\mathrm{N}_{j}^{3}\left(\xi_{k}, \eta_{k}, \zeta_{k}\right)\right]=\delta_{j k}
\end{array}
$$

Equation (21) may be written in the following form:

$$
\Psi_{\mathrm{i}}(\xi, \eta, \zeta)=\Phi_{\mathrm{r}}(\xi, \eta, \zeta)\left(\bar{\Psi}_{\mathrm{i}}\right)_{\mathrm{r}} \quad(\mathrm{r}=1,2 \cdots 32)
$$

where the interpolation functions $\Phi_{\mathrm{r}}$ are given in [2]

\section{COMPUTATIONAL PROCEDURE}

\subsection{Three-Dimensional Finite Element Formulation}

The fourth order partial differential equations in terms of the three-dimensional stream function vector components (equation (12)) may be written as the residual,

$$
\mathbf{R}=\frac{\partial}{\partial \mathrm{t}} \nabla^{2} \Psi+((\nabla \times \Psi) \cdot \nabla) \nabla^{2} \Psi-\left(\nabla^{2} \Psi \cdot \nabla\right)(\nabla \times \Psi)-v \nabla^{4} \Psi=0
$$

or using index notations,

$$
\mathrm{R}_{\mathrm{i}}=\frac{\partial}{\partial \mathrm{t}}\left(\Psi_{\mathrm{i}, \mathrm{j}}\right)+\varepsilon_{\mathrm{jkr}} \Psi_{\mathrm{k}, \mathrm{j}} \Psi_{\mathrm{i}, \mathrm{mm} r}-\varepsilon_{\mathrm{ski}} \Psi_{\mathrm{r}, \mathrm{j}} \Psi_{\mathrm{k}, \mathrm{sr}}-\nu \Psi_{\mathrm{i}, \mathrm{j} \mathrm{jk}}=0
$$

where the subscripts $i, j, k, m, r$, and s take the values 1,2 , and 3 .

The Generalized Galerkin finite element (GGFE) formulation of equation (25) in the combined spatial and temporal domain requires the successive inner product of the form [4],

$$
\left(\hat{\mathrm{W}}(\xi),\left(\Phi_{\alpha}, \mathrm{R}_{\mathrm{i}}\right)\right)=\int_{\xi} \hat{\mathrm{W}}(\xi)\left[\int_{\Omega} \Phi_{\alpha} \mathrm{R}_{\mathrm{i}} \mathrm{d} \Omega\right] \mathrm{d} \xi=0
$$

where $\Phi_{\alpha}$ and $\hat{W}(\xi)$ denote the spatial and temporal test functions, respectively, $\xi$ is the nondimensional temporal variable given by:

$$
\xi=\frac{\mathrm{t}}{\Delta \mathrm{t}}
$$

Assuming that the variation of $\Psi_{i}$ is approximated in the form: 


$$
\Psi_{\mathrm{i}}=\Phi_{\beta} \Psi_{\beta}
$$

we substitute Eqs. (25) and (28) into equation (26) and integrate the resulting equation by parts twice in the spatial domain to obtain:

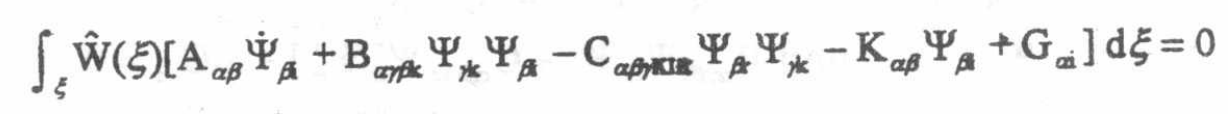

where

$$
\begin{aligned}
& \mathrm{A}_{\alpha \beta}=\int_{\Omega} \Phi_{\alpha, \mathrm{j}} \Phi_{\beta, \mathrm{j}} \mathrm{d} \Omega \\
& \mathrm{K}_{\alpha \beta}=\int_{\Omega} \nu \Phi_{\alpha, \mathrm{kk}} \Phi_{\beta, \mathrm{jj}} \mathrm{d} \Omega \\
& \mathrm{B}_{\alpha \gamma \beta \mathrm{k}}=\int_{\Omega} \varepsilon_{\mathrm{jkr}} \Phi_{\alpha} \Phi_{\gamma, \mathrm{j}} \Phi_{\beta, \mathrm{mmr}} \mathrm{d} \Omega \\
& \mathrm{C}_{\alpha \beta \mathrm{kir}}=\int_{\Omega} \varepsilon_{\mathrm{ski}} \Phi_{\alpha} \Phi_{\beta, \mathrm{j}} \Phi_{\gamma, \mathrm{sr}} \mathrm{d} \Omega \\
& \mathrm{G}_{\alpha i}=v \int_{\Gamma}\left(\Phi_{\alpha, \mathrm{k}}^{*} \Psi_{\mathrm{i}, \mathrm{j}} \mathrm{n}_{\mathrm{k}}-\Phi_{\alpha}^{*} \Psi_{\mathrm{i}, \mathrm{jk} \mathrm{k}} \mathrm{n}_{\mathrm{k}}\right) \mathrm{d} \Gamma
\end{aligned}
$$

Choosing a linear variation of the variables and their products in the temporal domain, such that:

$$
\begin{aligned}
& \Psi_{\beta}=(1-\xi) \Psi_{\beta}^{n}+\xi \Psi_{\beta k}^{n+1} \\
& \Psi_{\beta} \Psi_{j k}=(1-\xi) \Psi_{\beta}^{n} \Psi_{j k}^{n}+\xi \Psi_{\beta}^{n+1} \Psi_{j k}^{n+1}
\end{aligned}
$$

Substituting Eqs. (35) and (36) into equation (29) and performing the integration, we get:

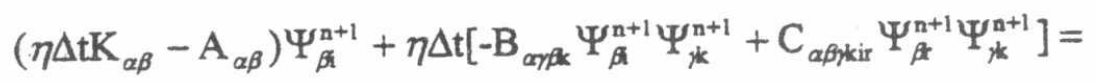

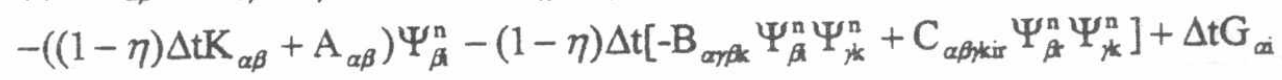

where $\eta$ is the temporal parameter defined as:

$$
\eta=\frac{\int_{\xi} \hat{\mathrm{W}}(\xi) \xi \mathrm{d} \xi}{\int_{\xi} \hat{\mathrm{W}}(\xi) \mathrm{d} \xi}
$$

with $\eta=1$ being used in this study for full implicitness. Equation (37) is the nonlinear finite element equations for the governing fourth order partial differential equations (12). 


\subsection{Newton-Raphson Method}

The nonlinear finite element equation (37), is rewritten in the form:

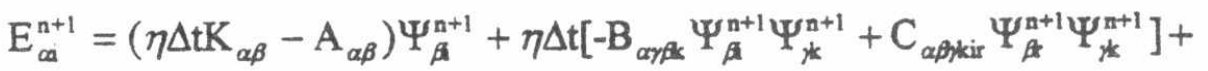

$$
\begin{aligned}
& \left((1-\eta) \Delta \mathrm{t} \mathrm{K}_{\alpha \beta}+\mathrm{A}_{\alpha \beta}\right) \Psi_{\beta}^{\mathrm{n}}+(1-\eta) \Delta \mathrm{t}\left[-\mathrm{B}_{\alpha \eta \boldsymbol{k}} \Psi_{\beta}^{\mathrm{n}} \Psi_{k \mathrm{k}}^{\mathrm{n}}+\mathrm{C}_{\alpha \beta \beta \mathrm{kir}} \Psi_{\beta}^{\mathrm{n}} \Psi_{\not k}^{\mathrm{n}}\right]-\Delta \mathrm{t} \mathrm{G}_{\alpha i}=0
\end{aligned}
$$

One of the most efficient approaches to solve nonlinear equations is the Newton-Raphson developed from Taylor series expansion of the residual, $E_{a i}$, of the type in equation (39).

$$
\mathrm{E}_{a i}^{\mathrm{n}+1, x+1}=\mathrm{E}_{\alpha i}^{\mathrm{n}+1, x}+\frac{\partial \mathrm{E}_{\alpha i}^{\mathrm{n}+1, x}}{\partial \Psi_{r i}^{\mathrm{n}+1, x}} \Delta \Psi_{r i}^{\mathrm{n}+1, x+1}+\cdots=0
$$

Equation (40) implies that the residual at a given time station $\mathrm{n}+1$ as incremented to the $\mathrm{r}+1$ cycle from the previous one should vanish for equation (39) to be satisfied. Retaining only the first order term in equation (40) yields

$$
\mathrm{J}_{a i n}^{\mathrm{n}+1, r} \Delta \Psi_{r j}^{\mathrm{n}+1, x+1}=-\mathrm{E}_{a i}^{\mathrm{n}+1, r}
$$

where $E_{o i}^{n+1, r}$ is the residual at the $r$ cycle(equation (39)),

$$
\Delta \Psi_{r i}^{n+1, r+1}=\Psi_{n j}^{n+1, x+1}-\Psi_{n}^{n+1, x}
$$

and $\mathrm{J}_{a \mathrm{x}, \mathrm{j}}^{\mathrm{n}+1, x}$ is the Jacobian given by:

$$
\mathrm{J}_{a i m p}^{\mathrm{n}+1, x}=\frac{\partial \mathrm{E}_{a}^{\mathrm{n}+1,5}}{\partial \Psi_{r j}^{\mathrm{n}+1,5}}
$$

Substituting equation (39) into equation (43), we obtain

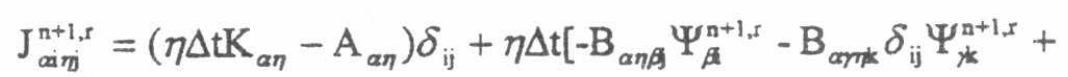

$$
\begin{aligned}
& \left.\mathrm{C}_{\alpha \beta \eta \mathrm{jk}} \Psi_{\beta k}^{\mathrm{n}+1, \mathrm{r}}+\mathrm{C}_{\alpha \eta \mathrm{kij}} \Psi_{\gamma k}^{\mathrm{n}+1, \mathrm{r}}\right]
\end{aligned}
$$

\subsection{Application of Boundary Condition}

The boundary conditions we may encounter in the use of the stream function vector formulation will have the following forms:

$$
\Psi_{\mathrm{i}}=0
$$




$$
\Psi_{1}-\Psi_{j}=0 \text { or } U \quad(i \neq j)
$$

where $i$, and $j=1,2 \ldots 12$ (12 is the number of unknowns per node).

Application of the boundary condition given by equation (45) is straight forward, since the type of element introduced in the preceding section has in addition to the variable its first derivatives. Such type of elements has the advantage that the boundary conditions involving the variable and its derivatives can be directly inserted into the element matrix as usually done with Dirichlet conditions. On the other hand, application of the boundary conditions given by equation (46) requires special attention especially with the nonlinear problem at hand. We will refer to these boundary conditions as the coupled boundary conditions. Two suitable ways to incorporate the coupled boundary conditions into the system of equations are presented in [2]. The first method will be presented in the global formulation of the finite element method and the second will be presented in the local level of the formulation. These boundary conditions for Laplace equation are discussed in [4] and [5], respectively.

The second method is to modify the element matrix with boundary conditions implemented directly. This modification is done to all element matrices that surround the boundary node under consideration. To impose such boundary conditions we first rewrites equation (41) in the local level as:

$$
\mathrm{J}^{(\mathrm{e})} \Delta \Psi^{(\mathrm{e})}+\mathrm{D}^{(\mathrm{e})} \Psi_{\text {old }}^{(\mathrm{e})}=-\mathrm{F}^{(\mathrm{e})}
$$

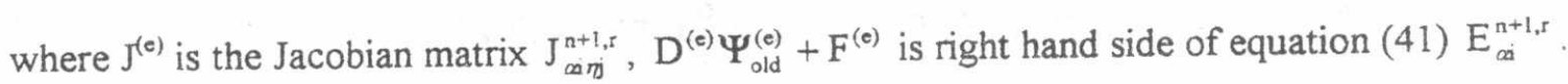

The dimension of the matrices in equation (47) is $96 * 96$ (96 is equal to the number of unknown per node multiplied by the maximum number of nodes per element). Then, to impose the coupled boundary conditions, equation (46), into the element equation matrices, equation (47), we proceed with the following procedure with both matrices $\mathrm{J}^{(e)}$ and $\mathrm{D}^{(e)}$ engaged unless otherwise noted.

1. For those elements that have the boundary node under consideration in common, the row corresponding to $\Psi_{i}$ is multiplied by 1 and added to the row corresponding to $\Psi_{j}$.

2. To effect the substitution of $\Psi_{i}=\Psi_{j}+U$ in the system of equations the column corresponding . to $\Psi_{1}$ is multiplied by 1 and added to the column corresponding to $\Psi_{j}$, and the column corresponding to $\Psi_{i}$ in the $D^{(e)}$ matrix is multiplied by $U$ and subtracted from the right hand-side $F^{(e)}$

3. The row and column corresponding to $\Psi_{\mathrm{i}}$ is replaced by zeros.

4. To eliminate the zero on the diagonal of the $\Psi_{i}$ equation arising in the previous step, simply insert the condition $\Psi_{\mathrm{i}}-\Psi_{\mathrm{j}}=U$ directly in the row corresponding to $\Psi_{\mathrm{i}}$ by entering 1 and -1 in the column corresponding to $\Psi_{i}$ and $\Psi_{j}$, respectively and insert $U$ in the right hand-side.

This procedure incorporates the coupled condition equation (46) as one of the system of equations with the necessary modification as described above. However, it is noted that this method makes the system of matrices nonsymmetric, which is often a disadvantage. 
Since the system of matrices given in equation (41) is already nonsymmetric, we have adopted the second method in our computations.

\subsection{Physical Model}

From the numerical viewpoint, three-dimensional flows in cavities serve as ideal prototype for testing numerical codes. Some of the attractive features of the cavity flow are its simple geometry, its simple boundary conditions, its recirculating nature, and the availability of numerical results by other investigators. In the cubic cavity the flow is driven by the uniform translation of one of its walls while all other walls are held at rest. Due to the symmetry of the three-dimensional cavity flow, see for example [6], only one-half of the computational domain needs to be solved.

In the present investigation the cubic cavity flow is assumed to be steady, laminar and incompressible Newtonian fluid, with constant properties. So Eqs. (25), (37), (39), and (44) will take the following forms, respectively.

$$
\begin{aligned}
& \mathrm{R}_{\mathrm{i}}=\varepsilon_{\mathrm{j} k r} \Psi_{\mathrm{k}, \mathrm{j}} \Psi_{\mathrm{i}, \mathrm{mmr}}-\varepsilon_{\mathrm{ski}} \Psi_{\mathrm{r}, \mathrm{jj}} \Psi_{\mathrm{k}, \mathrm{sr}}-\nu \Psi_{\mathrm{i}, \mathrm{j} \mathrm{jk}}=0 \\
& \mathrm{~B}_{\alpha \gamma \beta \mathrm{k}} \Psi_{\gamma k} \Psi_{\beta \mathrm{k}}-\mathrm{C}_{\alpha \beta \gamma \mathrm{kir}} \Psi_{\beta \mathrm{k}} \Psi_{\gamma k}-\mathrm{K}_{\alpha \beta} \Psi_{\beta k}+\mathrm{G}_{\alpha i}=0 \\
& \mathrm{E}_{\alpha i}=\mathrm{B}_{\alpha \gamma \beta \mathrm{k}} \Psi_{\gamma k} \Psi_{\beta \boldsymbol{k}}-\mathrm{C}_{\alpha \beta \gamma \mathrm{kir}} \Psi_{\beta k} \Psi_{\gamma k}-\mathrm{K}_{\alpha \beta} \Psi_{\beta \boldsymbol{k}}+\mathrm{G}_{\alpha i}=0 \\
& \left.\mathrm{~J}_{\alpha i \eta j}=\mathrm{K}_{\alpha \eta} \delta_{\mathrm{ij}}-\mathrm{B}_{\alpha \eta \beta \xi} \Psi_{\beta \boldsymbol{k}}-\mathrm{B}_{\alpha \eta \eta \mathrm{k}} \delta_{\mathrm{ij}} \Psi_{, \mathrm{k}}+\dot{\mathrm{C}}_{\alpha \beta \eta \mathrm{ik}} \Psi_{\beta \mathrm{k}}+\mathrm{C}_{\alpha \eta / \mathrm{kij}} \Psi_{, k}\right]
\end{aligned}
$$

Fig. 3 shows the geometry considered in the numerical simulation of the cubic cavity of length $\mathrm{L}=1.0 \mathrm{~m}$. The motion of the cavity is driven by the top wall with constant unit velocity, $\mathrm{U}_{\max }=1.0$ $\mathrm{m} / \mathrm{s}$, in the positive $\mathrm{x}$-direction, while the other surfaces remain stationary with the boundary conditions as shown in Table 1.

\section{COMPUTATIONAL RESULTS}

The eight corner nodes hexahedral element with four variables per node (see Fig. 2) has been adopted in our computations. The numerical integration using Gaussian-qudrature has been tested with the number of Gaussian points ranging from 2 to 5 , showing that a complete convergence occurs at four points as shown in Fig. 4. The calculations were terminated when the solution vector obtained at the end of two successive Newton-Raphson iterations differs by a small number. The convergence criteria used in the computation are in the form

$$
\begin{aligned}
& \mathrm{E} 1=\max (\Delta \Psi) \leq \varepsilon \\
& \mathrm{E} 2=\left(\frac{\sum_{i=1}^{N}\left|\Psi_{i}^{\mathrm{r}+1}-\Psi_{i}^{\mathrm{r}}\right|^{2}}{\sum_{\mathrm{i}=1}^{N}\left|\Psi_{i}^{\mathrm{r}+1}\right|^{2}}\right)^{1 / 2} \leq \varepsilon
\end{aligned}
$$


where $\mathrm{N}$ is the total number of unknowns in the finite element mesh, $\Psi_{\mathrm{i}}^{\mathrm{r}}$ solution unknown at node $\mathrm{i}$ and $\mathrm{rth}$ iteration, and $\varepsilon=10^{-5}$. Only a few cycles were need to obtain convergence. In addition to the above criteria, the RMS error of the residual $\mathbf{R}$, equation (39), is monitored during the computation and it is found that its value is reduced by 10 orders of its original value when convergence is reached.

\section{Table1. Boundary Conditions (3-D Cavity)}

\begin{tabular}{|c|c|}
\hline & $\Psi_{1,1}=\Psi_{2}=\Psi_{2,2}=\Psi_{2,3}=\Psi_{3}=\Psi_{3,2}=\Psi_{3,3}$ \\
& $\Psi_{1,3}-\Psi_{3,1}=0, \Psi_{2,1}-\Psi_{1,2}=0$
\end{tabular}

Computations are performed for $\operatorname{Re}=10$, and $\operatorname{Re}=100$ using a uniform grid of $11^{*} 11^{*} 6$ and $15 * 15 * 8$, respectively. The computations of the three-dimensional cavity were performed on the C-90 Cray machine. Approximately 11 megabytes of core is required for these simulations with the $11^{*} 11^{*} 6$ mesh, while 31 megabytes is required with the $15 * 15 * 8$ mesh. Table 2 shows the average CPU time per one iteration for the solution of the 3-D lid-driven cavity.

Table 2. CPU Time per One Iteration (3-D Cavity)

\begin{tabular}{|c|c|c|c|c|}
\hline $\begin{array}{c}\text { Mesh } \\
\text { Size }\end{array}$ & Re & $\begin{array}{c}\text { Average CPU per one } \\
\text { iteration for Forming } \\
\text { System Matrices (sec) }\end{array}$ & $\begin{array}{c}\text { Average CPU per one } \\
\text { iteration for GMRES } \\
\text { Solver (sec) }\end{array}$ & $\begin{array}{c}\text { Total Average CPU per } \\
\text { one iteration (sec) }\end{array}$ \\
\hline $11^{*} 11^{*} 6$ & 10 & 77 & 52 & 129 \\
\hline $11^{*} 11^{*} 6$ & 100 & 77 & 92 & 169 \\
\hline $15^{*} 15^{*} 8$ & 100 & 210 & 495 & 705 \\
\hline
\end{tabular}


Fig. 5 presents velocity profiles along the vertical centerline of the cubic cavity for $R e=100$. In this figure, the solid line represents the present prediction $(15 * 15 * 8$ finite element mesh) compared with Goda [7] $(20 * 10 * 20$ finite difference mesh using the velocity-pressure formulations), Mahallati and Militzer [8] $(21 * 11 * 21$ finite difference mesh using the vorticityvector potential formulations), and Takami and Kuwahara [9] (20*10*20 finite difference mesh using velocity pressure formulations). It is seen that agreement with these results is reasonable. The effect of mesh size on the velocity profile along vertical and $x$-horizontal centerlines of the 3$\mathrm{D}$ cavity for $\mathrm{Re}=100$ is shown in Fig. 6 indicating that convergence has already been reached with the coarse mesh of $11^{*} 11^{*} 6$. The rapid convergence of the solution for $\operatorname{Re}=10$, and $\operatorname{Re}=$ 100 is shown in Fig. 7. It is seen that the required accuracy is obtained in a few iterations.

Fig. 8 shows the $x$ =component of the velocity profiles of $R e=100$ at the $x=0.5$ and $x=0.786$ planes, respectively. It is seen that the velocity profiles in planes close to the wall are less developed due to the boundary layer effects than that in the symmetry plane. Goda [7], and Mahallati and Militzer [8] present similar profiles at the symmetry plane of the cubic cavity for $\mathrm{Re}$ $=100$. It is difficult to compare their graphs directly with the present ones, but a visual inspection indicates that all agree very well.

For the 3-D cavity flow the streamlines at the symmetry plane $(z=0.5)$, and the $z=0.2$ plane are shown in Fig. 9. It is noted that, for higher Reynolds number $(\mathrm{Re}=100)$, the vortex center moves in the downstream direction with the position of the vortex center for $\operatorname{Re}=100$ at the $z=0.2$ plane is close to the moving boundary than that at the symmetry plane $(z=0.5)$, as expected. Fig. 10 shows the velocity profiles along the vertical and horizontal centerlines of the symmetry plane of the 3-D cavity. It is seen that, in Fig. 10a, an increase in Reynolds number tends to reduce negative xvelocity in the region around $y=0.6$, with the point of maximum negative $x$-velocity moving downward. At the same time, the y-velocity becomes less positive upstream and more negative downstream as the Reynolds number increases, with the position of zero-velocity shifted toward downstream as shown in Fig. $10 \mathrm{~b}$.

\section{CONCLUSIONS}

The vorticity transport for three-dimensional incompressible flow has been recast into the fourth order partial differential equations in terms of the three stream function vector components. The finite element formulations with new interpolation functions and Newton-Raphson iterations led to excellent computational results. Although computations are expensive there are several significant advantages which make the present approach extremely attractive. Higher order finite element interpolation functions corresponding to the fourth order partial differential equations are capable of producing very stable and convergent solutions. Furthermore, boundary condition implementation is much simpler and versatile as compared with other currently available methods. Finally, accuracy achieved in the proposed scheme opens the door to many three-dimensional flows, particularly in acoustic or unstable wave problems and most importantly the transition toward turbulence, by constructing the three-dimensional version of Orr-Sommerfeld type eigenvalue problems. These projects are currently under progress. 


\section{REFERENCES}

[1] Chung, T. J., Applied Continuum Mechanics. Cambridge University Press, 1996.

[2] Elshabka, A. M., Existence of three-dimensional stream function vector components and their applications in three-dimensional flow. $\mathrm{Ph}$. D. Dissertation. The University of Alabama in Huntsville, Al., 1995.

[3] Elshabka, A. M. and Chung, T. J., New Definition of Three-Dimensional stream Function Vector, Verification of Theory, and Associated Physics. 1st AIAA Theoritical Fluid Mechanics Meeting. New Orleans, LA, June 17-20, 1996.

[4] Chung, T. J., Computational Fluid Dynamics, Lecture Notes, Department of Mechanical and Aerospace Engineering, UAH, 1995.

[5] Norrie, D. H. and de Vries, G., An Introduction to Finite Element Analysis, Academic Press, 1978.

[6] Kosef, J. R., Street, R. L., Gresho, C. D., Upson, C. D., Humphrey, J. A. C., and To, W. -M., A Three-Dimensional Lid-Driven Cavity Flow: Experiment and Simulation, Proc. 3rd Int. Conf. Num. Meth. Lam. and Turb. Flow, Seattle, 1983.

[7] Goda, K., A Multistep Technique with Implicit Difference Schemes for Calculating Two- or Three-Dimensional Cavity Flows, Journal of Computational Physics, 1979, Vol. 30, 76-95.

[8] Mahallati, A. and Militzer, J., Application of the Piecewise Parabolic Finite Analytic Method To the Three-Dimensional Cavity Flow, Numerical Heat Transfer, 1993, Vol. 24, Part B, 337-351.

[9] Takami, H. and Kuwahara, K., Numerical Study of Three-Dimensional Flow within a Cavity, Journal of Physical Society of Japan, 1974, Vol. 73, No. 6, 1695-1698. 


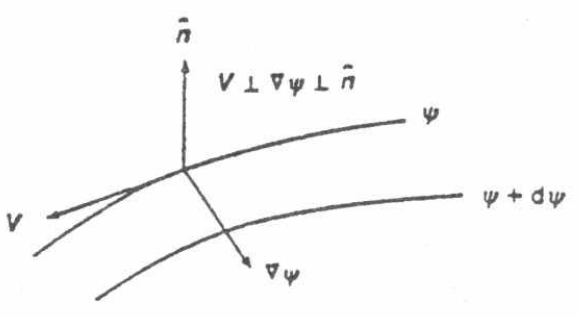

(a)

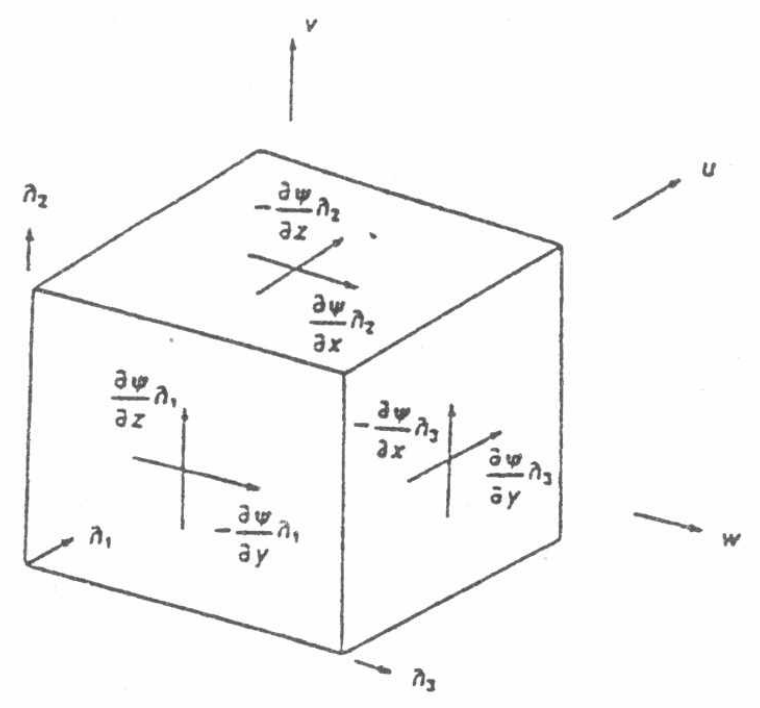

(b)

FIG. 1. General Description of Three-Dimensional Stream Functions

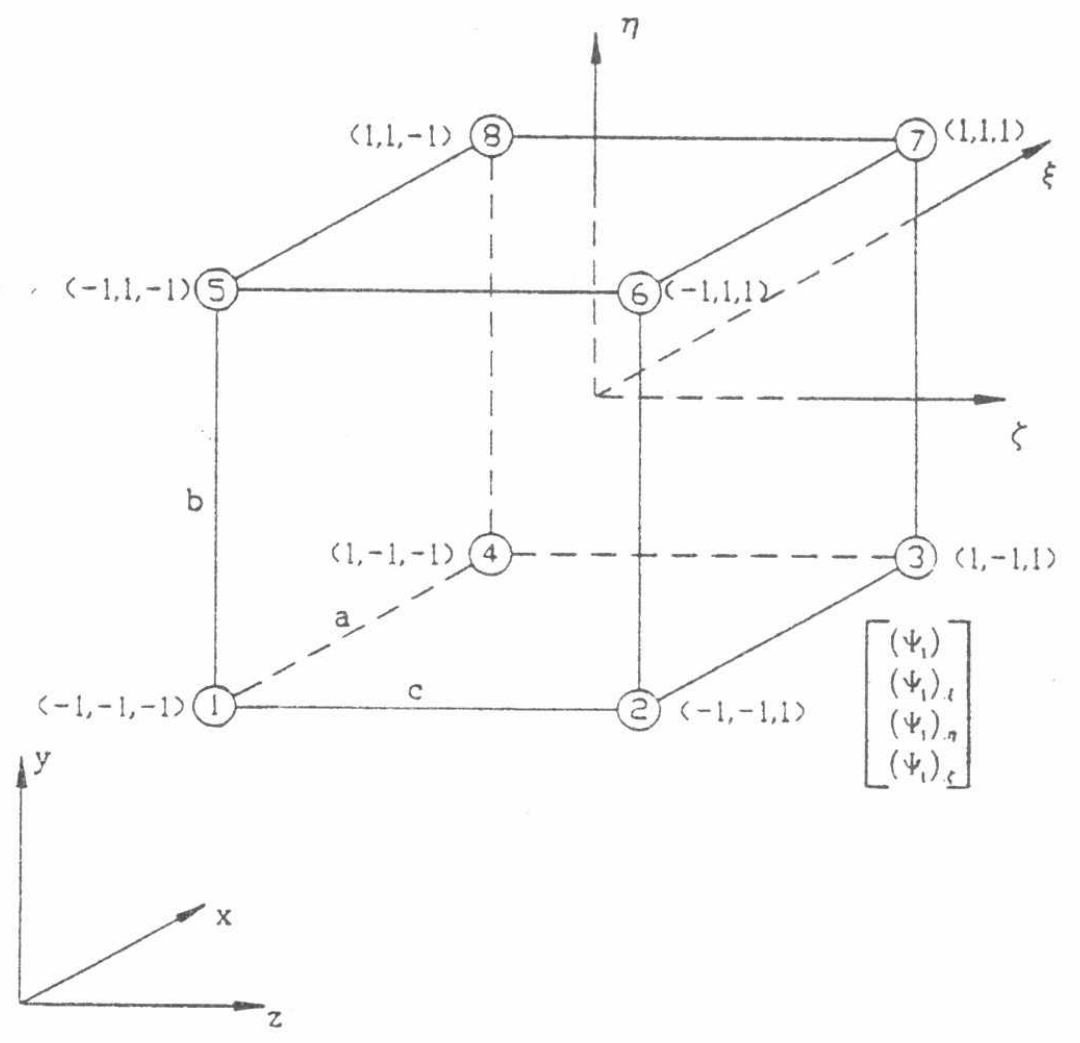

FIG. 2. Hexahedral Element 


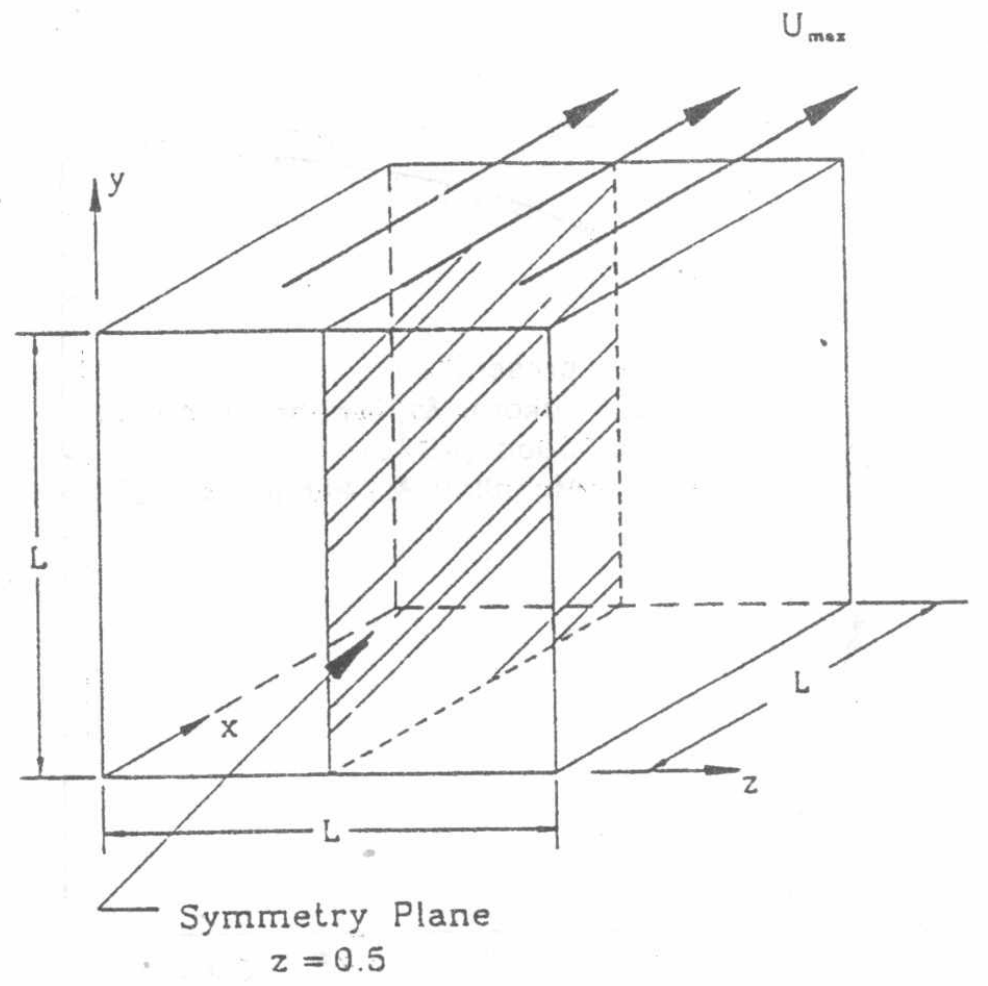

FIG. 3. Geometry of the Cubic Cavity Flow

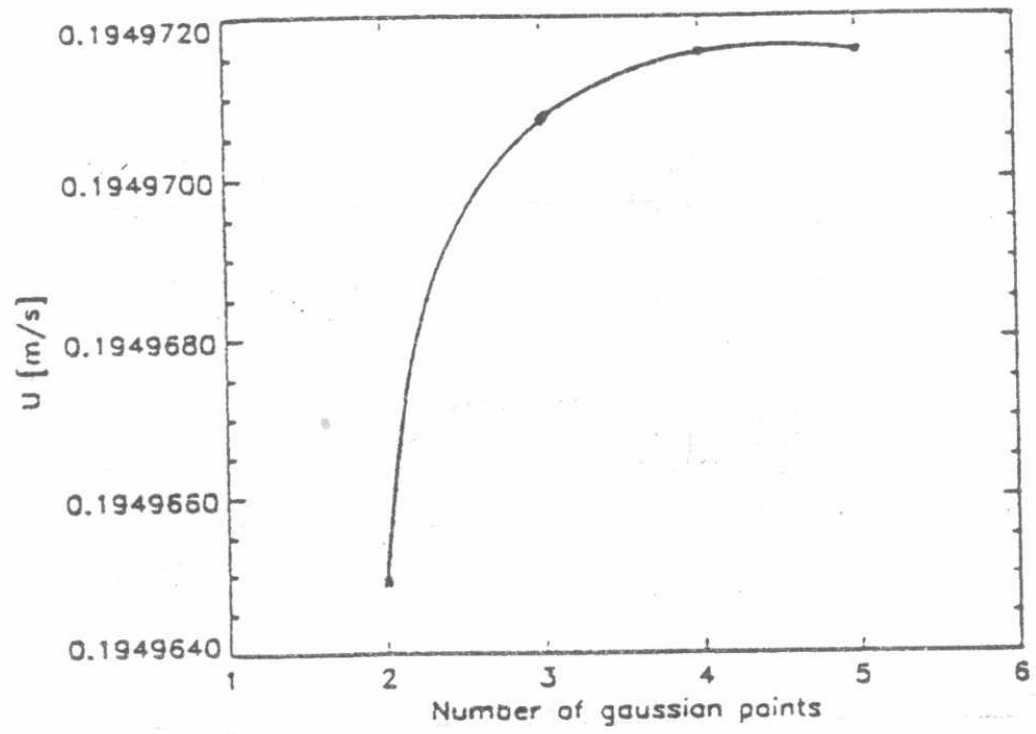

FIG. 4. X-Component of the Velocity Vector at the 3-D Cavity Center for $\mathrm{Re}=10$ Versus Number of Gaussian Points 


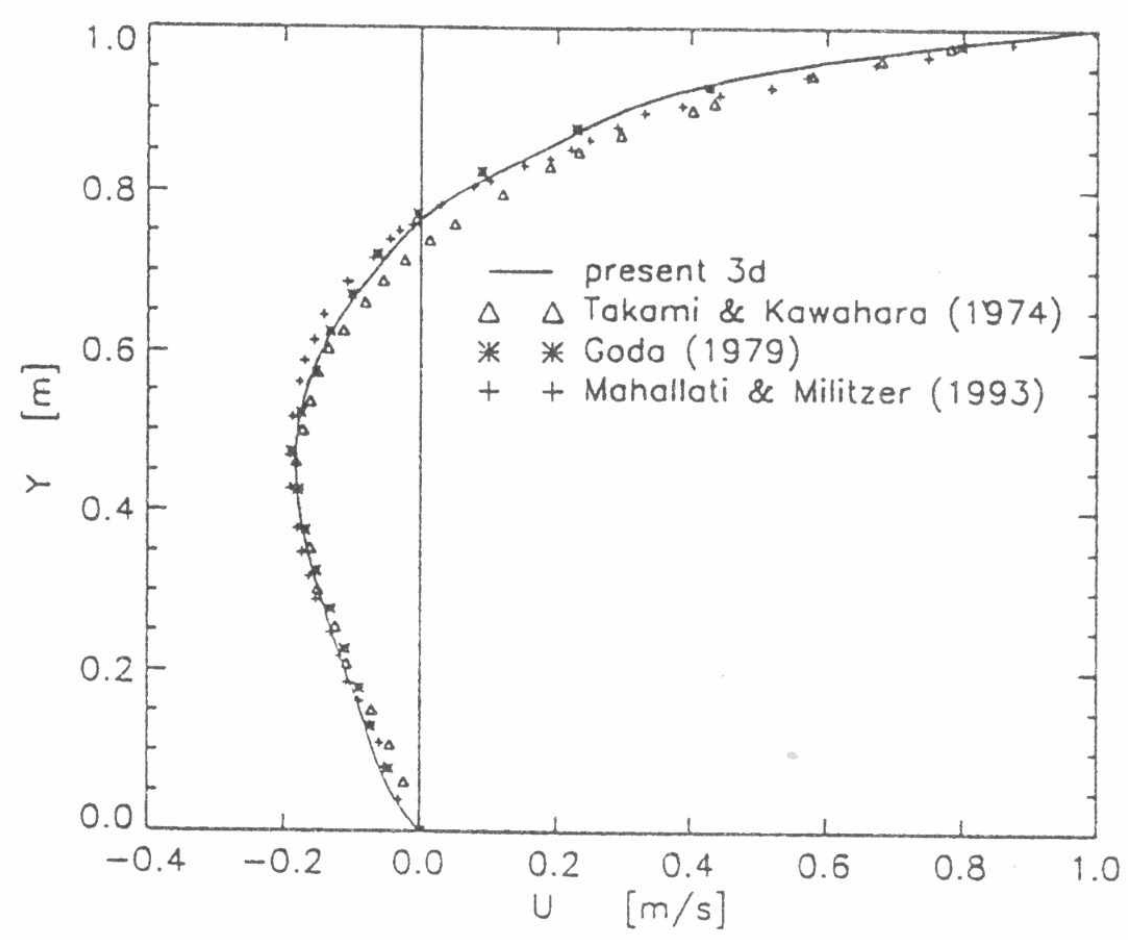

FIG. 5. Velocity profiles on vertical centerline of the 3-D cavity for $\mathrm{Re}=100$.

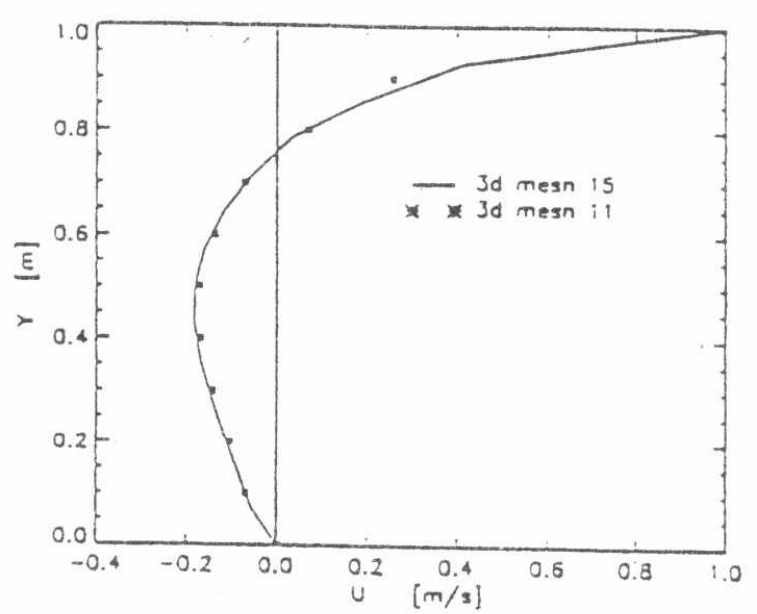

(a)

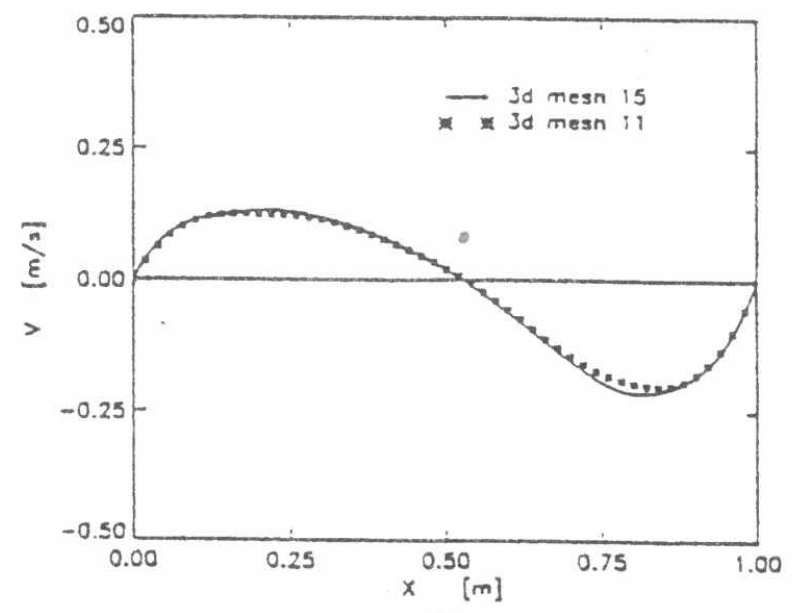

(b)

FIG. 6. Effect of Mesh Size for Re $=100$ on the 3-D Caviry: (a) Vertical Centeriine; (b) XHorizontal Centerline 


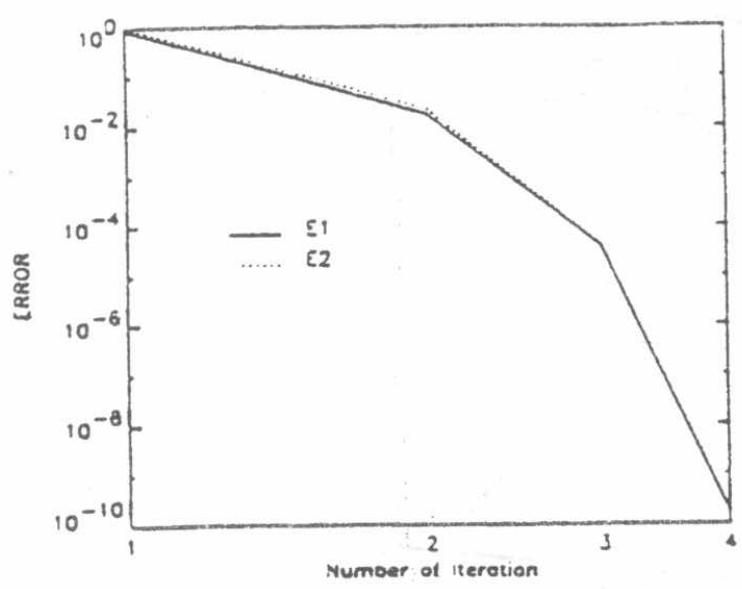

(a)

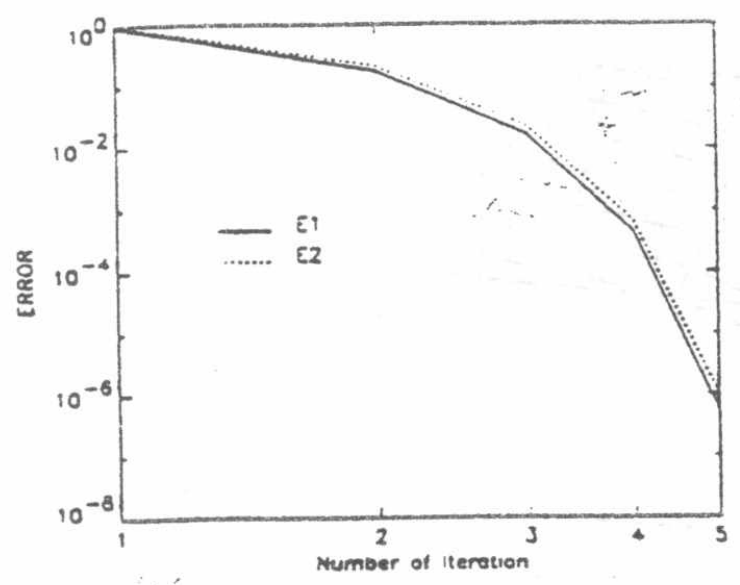

(c)

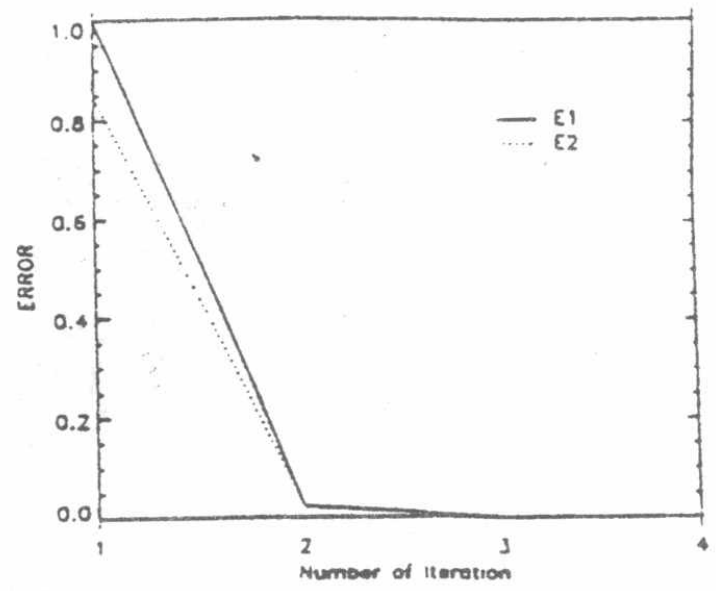

(b)

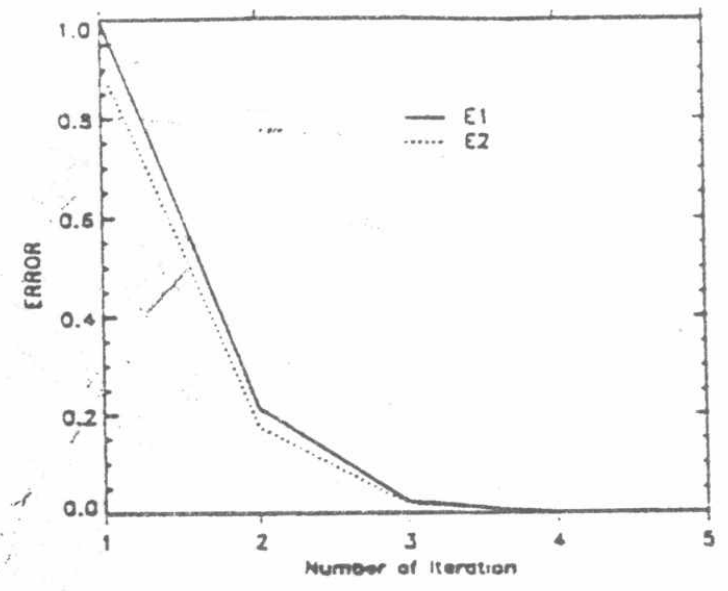

(d)

FIG. 7. Convergence History of the Solution of the 3-D Cavity for: (a) $\operatorname{Re}=10$, Mesh $11^{*} 11^{*} 6$, Logarithmic Scale; (b) Ré = 10, Mesh 11*11*6, Arithmetic Scale; (c) Re = 100, Mesh $15 * 15 * 8$, Logarithmic Scale; (d) Re $=100$, Mesh 15*15*8, Arithmetic Scale

$$
E 1=\max (\Delta \Psi) \leq 10^{-5} ; \quad E 2=\left(\frac{\sum_{i=1}^{N}\left|\Psi_{1}^{r-1}-\Psi_{i}^{r}\right|^{2}}{\sum_{i=1}^{N}\left|\Psi_{i}^{r-1}\right|^{2}}\right)^{1 / 2} \leq 10^{-5}
$$



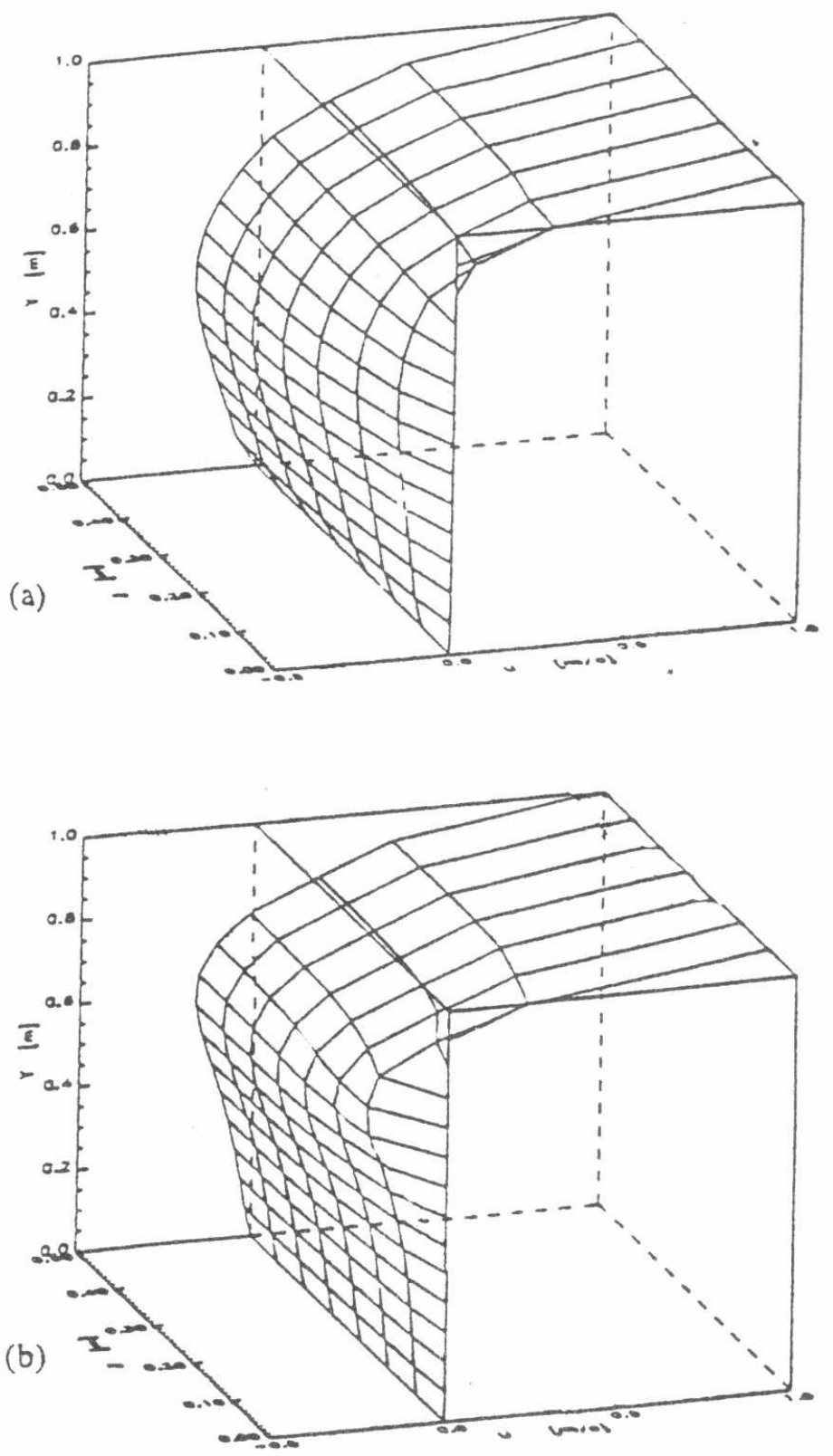

FIG. 8. Profiles of the X-Component of the Velocity for the 3-D Cavity flow of $R e=100$ at: (a) the $X=0.5$ Plane; $(b)$ the $X=0.786$ Plane. 

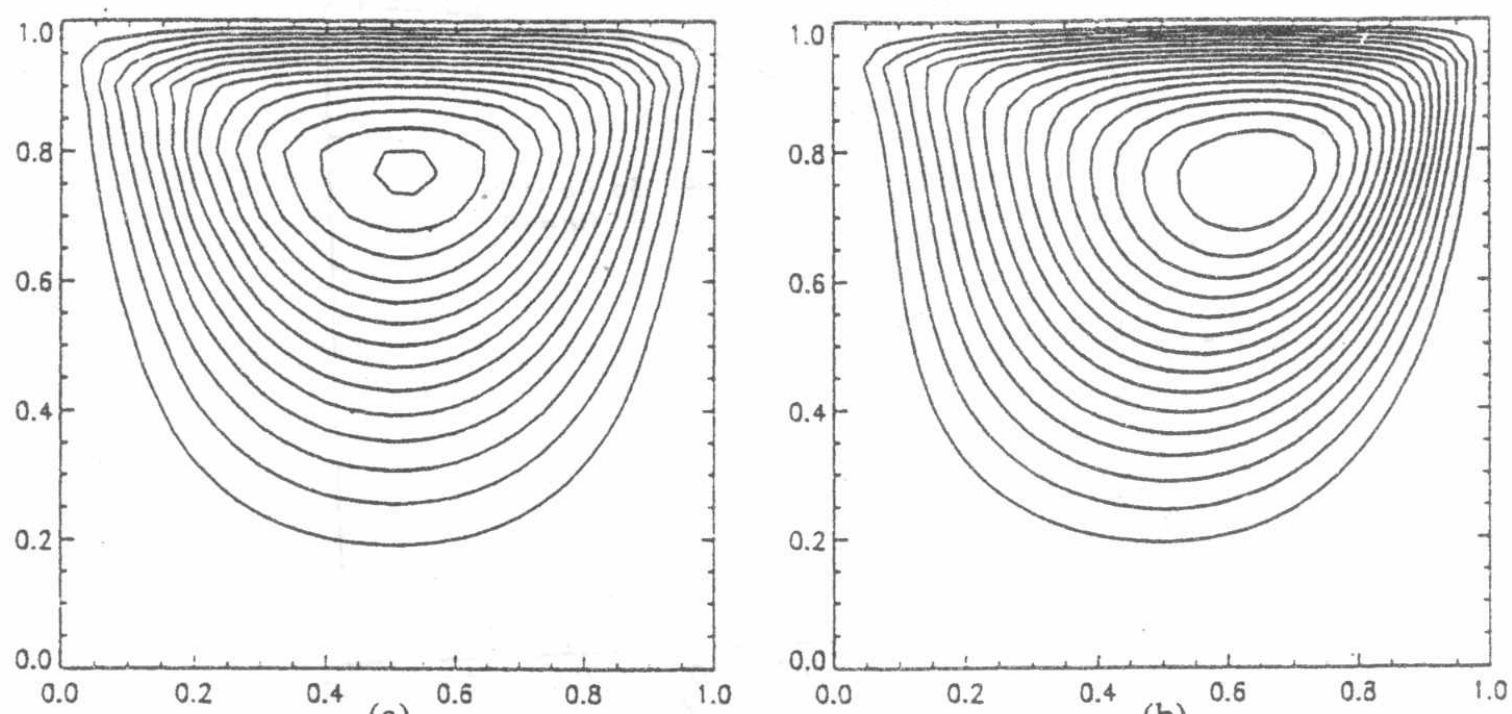

(a)

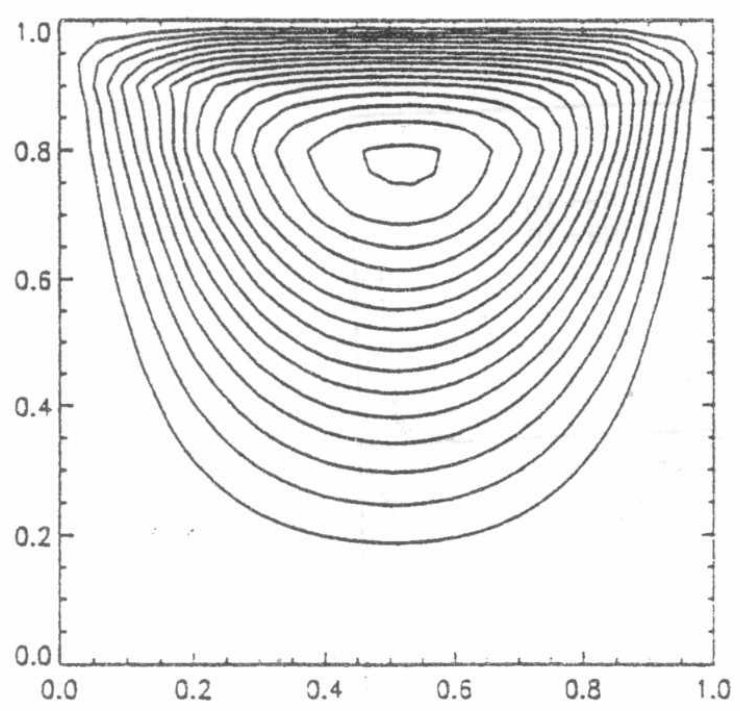

(c)

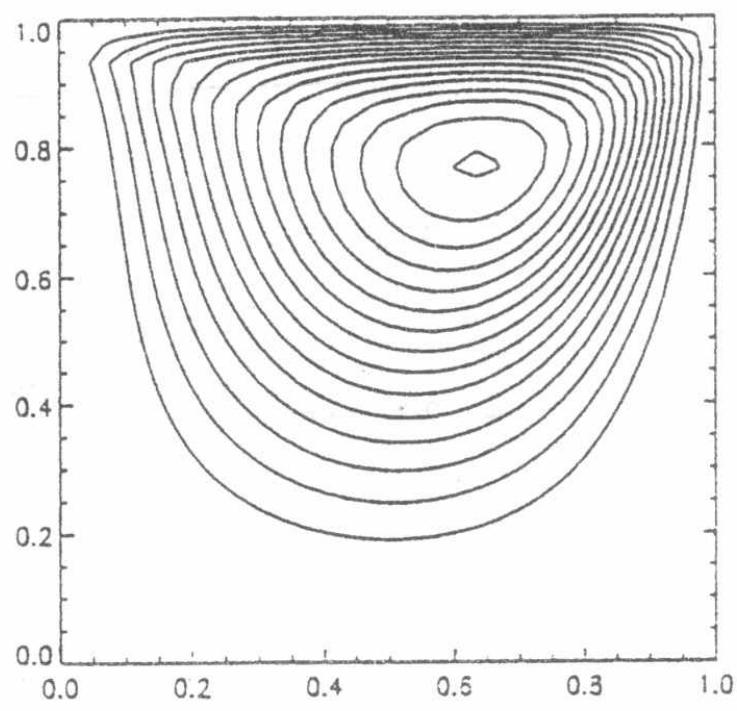

(d)

FIG. 9. The 3-D cavity streamlines $\left(\Psi_{3}\right)$ at (a) the symmetry plane $(z=0.5)$ for $\operatorname{Re}=10$, (b) the symmetry plane $(z=0.5)$ for $\operatorname{Re}=100$, (c) the $z=0.2$ plane for $\operatorname{Re}=10$, (d) the $z=$ 0.2 plane for $\operatorname{Re}=100$. 


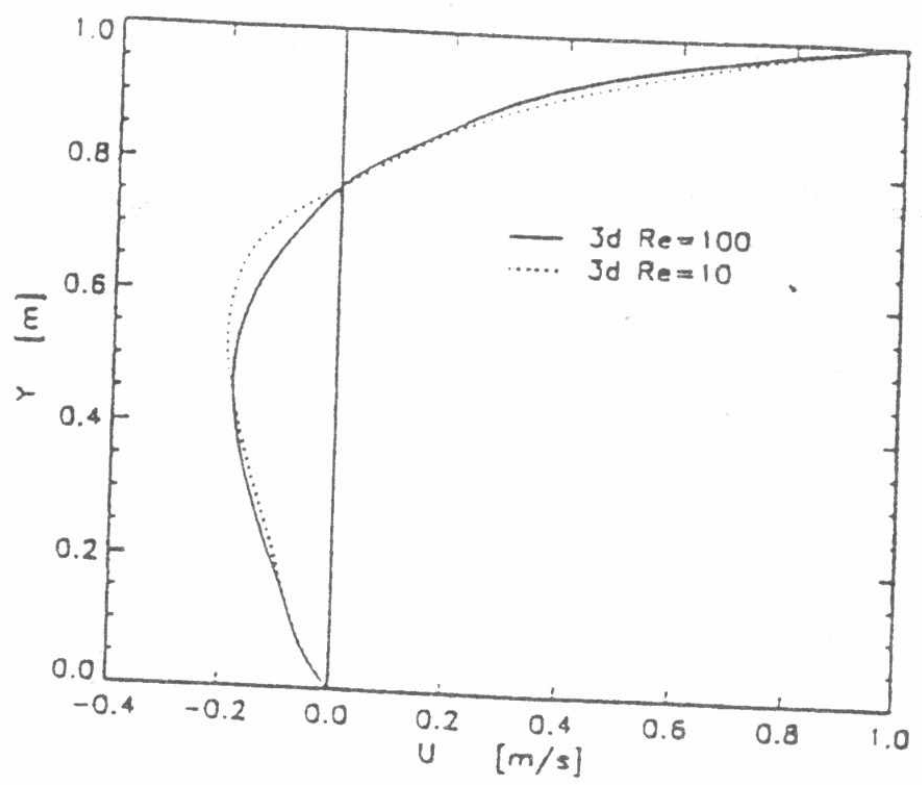

(a)

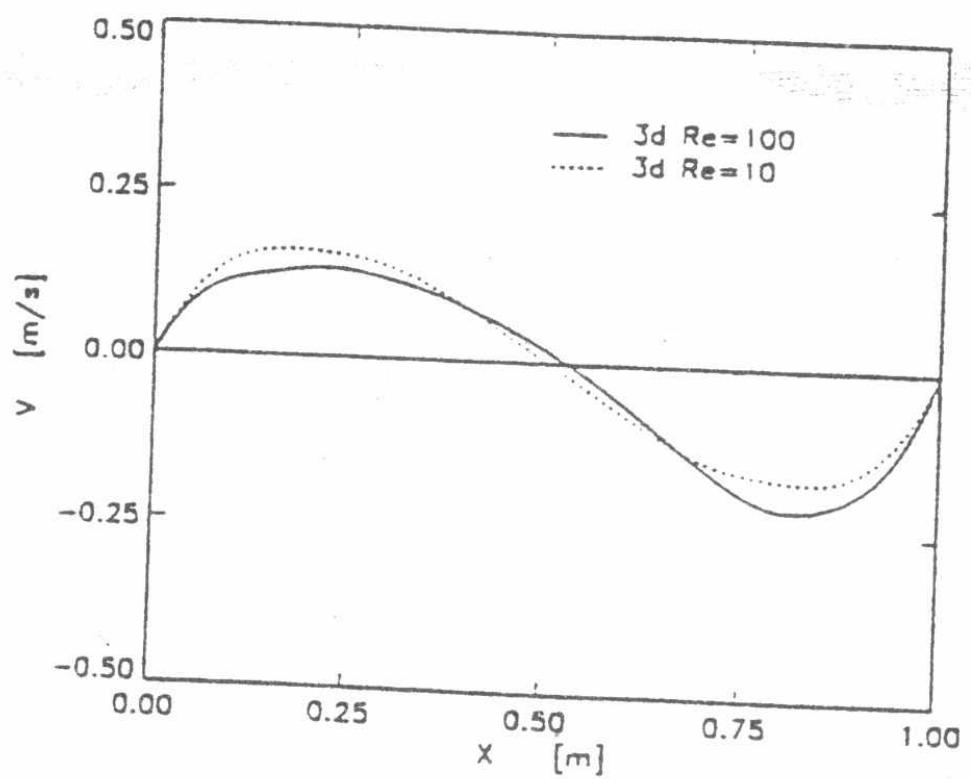

(b)

FIG. 10. Velocity Profile on the 3-D Cavity: (a) Vertical Centerline: (b) X-Horizontal Centerline 